\title{
Tröstliche Tode
}

Ottmar Leidner

Als Neurologe und Psychiater arbeite ich vorwiegend in der Rehabilitationsmedizin, also mit Menschen, die in der Aufgabe stehen, Unversehrtheit zu verabschieden und unerwartete neue Kapitel ihrer Biographie zu entwerfen. Zugleich bin ich als Arzt auch immer wieder mit dem Tod konfrontiert. Das Sterben beschäftigt mich seit Kindheit anhaltend und hat schon meine Berufswahl beeinflusst. Der Tod ist für die, denen er einen geliebten Menschen raubt, schwerste Belastung. Und für viele Sterbende ist er die größte Herausforderung ihres Lebens. Die Gewissheit unseres Todes kann als unerträglich erlebt werden. Aber sie kann auch Anlass sein, sich auf die Suche $\mathrm{zu}$ machen und $\mathrm{zu}$ fragen, wie ein liebender Gott denn damit umgehen will. Wenn diese schwere und letzte Aufgabe unvermeidlich zum Leben gehört - wie kann Gott dann seine $\mathrm{Zu}-$ sage einlösen, uns darin beizustehen? Manchmal scheint in einem Sterben ein Funke davon auf und bringt Hoffnung. Das zeigen auch die folgenden Sterbenarrative.

Sie wurden so weit anonymisiert, dass sie nicht mit wenigen Mausklicks zu entschlüsseln sind. Sollten Leser die Betroffenen durch Zufall kennen, wird um Diskretionsschutz gebeten. Ohne Einblicke ins sehr Private lassen sich solche Lebens- und Sterbegeschichten nicht weitergeben.

\section{1.}

S. war ein von vielen sehr geschätzter Arzt. Seine Kollegen und Vorgesetzten achteten seine Sorgfalt und Sachkenntnis, aber auch seine kollegiale Warmherzigkeit, die ein klares Wort nicht ausschloss. Seine Patienten spürten, wie gründlich und nimmermüde er sich um Diagnose und Therapie kümmerte. Ihr Wohl ging ihm erkennbar über fast alles andere.

In seiner Fachgesellschaft übernahm er wichtige Ämter, die er im Zugehen auf den Ruhestand geordnet übergab. Aber den Vordergrund, die große Bühne, suchte er nie. Viele waren deshalb überrascht, als er zu seinem Amtsabschied eine Rede hielt. Sie war ruhig, sachlich und von humanistischem Geist geprägt. Aber wer ihn näher kannte, konnte sehr kritische Aussagen über die Entwicklung des 
Gesundheitswesens mit zunehmendem ökonomischem Primat und abnehmendem Einfluss ärztlichen Denkens und Priorisierens heraushören, mahnend auch an die ärztlichen Leistungserbringer selbst.

Wir applaudierten schließlich stehend, und es wurde geraunt: "Was macht er da? Das ist doch mehr als eine Übergabe, das ist ein Vermächtnis. «Die meisten von uns wussten nicht, sondern erfuhren erst später, dass er sich zu dieser Zeit schon mit Herzbeschwerden herumschlug und Abklärung in die Wege geleitet hatte.

An diesem Abend führten wir beim Wein ein langes Gespräch über die Zukunft des Arztseins, bei dem aber auch klar wurde, dass seine Verwurzelung im christlichen Glauben viel tiefer war, als er öffentlich zur Sprache brachte. In einer weitgehend säkularen Umgebung wählte er wie manche andere den Weg, einerseits an seiner aktiven Zugehörigkeit zur (evangelischen) Kirche keinen Zweifel zu lassen, andererseits aber Glaubensäußerungen zu vermeiden, die als selbstgewiss missverstanden werden und ausgrenzen oder provozieren könnten.

Wenig später kam die Nachricht, dass er plötzlich verstorben war, am Feiertag Christi Himmelfahrt.

Er hatte mit seiner Frau und einem befreundeten Paar eine Wanderung vereinbart und beim Schließen der Haustür um Verständnis gebeten - er wolle sich nur noch eine Minute hinlegen, ihm sei nicht wohl. Wenige Minuten später war klar, dass es um Leben und Sterben ging, und seine Begleiter reanimierten. Vergeblich.

Das Leid der Angehörigen war arg. Aber die Pointe war für Glaubende nicht zu übersehen: Ein integrer und zeit seines Lebens dem Guten dienender Mann wurde ohne Siechtum und langes Elend mitten aus dem Leben heimgeholt. Am Himmelfahrtstag. In Wanderstiefeln. Der Tod ist und bleibt hart. Aber vielleicht misst der Herr über Leben und Tod anders und wählt manchmal Bilder und Szenen, die man in einem Film oder auf der Bühne für sehr zugespitzt halten würde.

\section{2.}

Spät abends kam der Anruf einer befreundeten Krankenschwester, deren drittes Kind in diesen Tagen geboren werden sollte: »Ich glaube, ich habe ein ganz, ganz krankes Kind zur Welt gebracht." Schon der allererste Befund wies auf ernsten Sauerstoffmangel und binnen weniger Stunden war klar, dass anatomische Anomalien am 
Herzen notfallmäßige Operationen erfordern. Andernfalls wäre ein Überleben und Gedeihen nicht denkbar.

Die ersten Lebensmonate waren also durch mehrere Operationen am offenen Herzen geprägt und entsprechend dramatisch. Die Aussichten auf eine snormale Entwicklung gingen gegen Null, und bald wurde klar zur Herztransplantation geraten, mit der Aussicht, diese bei einem wachsenden Kind im Lauf der Entwicklung mehrfach wiederholen zu müssen.

Wider alles Erwarten entwickelte sich das Mädchen trotz eines katastrophalen Ultraschallbefunds am Herzen körperlich, geistig und psychisch so, dass die Entscheidung zur Herztransplantation immer wieder aufgeschoben wurde. Sie wurde getauft, und es wurde viel über die richtigen Schritte nachgedacht, gesprochen und im Stillen wohl auch viel gebetet. Sie war stresssensibel. Bei Unruhe ließ sie sich als Säugling fast nur in der Wippe beruhigen, mit einer Wippfrequenz um 120-160/Minute. Man mochte sich das allenfalls über ihre eigene Ruheherzfrequenz und die Stresserinnerung des kleinen Körpers erklären.

Sie lernte gehen und sprechen, nur führte jede körperliche Anstrengung zu schwerer Atemnot. Nach langem Abwägen fand sich ein Kindergarten, der das Kind aufnahm, mit dem offen besprochenen Risiko jederzeitigen Sekundenherztods. Alle sahen, wie schwer es einem Vorschulkind fiel, nicht mit den Anderen tollen und rennen zu können. Aber alle akzeptierten dieses besondere, geschwächte, gehandicapte Kind, das überdies bald durch seltsame Sätze auffiel. Mit fünf Jahren bat sie ihre Mutter, die häufig zu offener Rede über Jugend und Alter, über Werden und Vergehen herausgefordert war: "Erzähl mir doch noch mal die Geschichte von wenn ich tot bin!»

Damals stand auch die Frage an, welche Schule damit fertig werden würde, ohne Unterforderung und ohne Überforderung, ohne Schulsport und mit allen Risiken. Das Mädchen schien trotz schweren körperlichen Handicaps geistig und psychisch absolut in der Lage, eine snormale< Schule zu meistern. Sie war wissbegierig, konnte zuhören, nahm schnell auf und hatte die typischen Wünsche ihres Alters. Ihr drängendster Ärger war damals, dass sie nicht wie ihre Altersgenossen ohne Stützräder Fahrrad fahren konnte.

Nur die gesundheitliche Ungewissheit und die Chancen im Wetteifern, auf das Erobern eines Platzes in einer nicht immer rücksichtsvollen Umgebung, konnten Sorgen machen und weit vorausgeworfen der Blick auf Pubertät und Adoleszenz, auf die Chancen in der Berufs- und Partnerwahl. 
Es kam anders: Sie übte und übte auf dem Fahrrad, und eines Tages gelang es ihr, wie ihr gleichaltriger Freund, ohne Stützräder eine lange Strecke ohne Absetzen und Sturz zu bewältigen. Sie stieg $a b$, jubelte, fiel um und war tot.

Niemand versuchte, sie zu reanimieren. Erstens war es fest so vereinbart. Zweitens: Wer darf schon mit einem Jubel auf den Lippen und im Herzen sterben? Das Begräbnis mit mehreren Hundert Trauernden war sehr bewegend, aber viele fühlten und sprachen aus: Es hatte auch etwas von einem Fest.

\section{3.}

M. hatte vier Kinder großgezogen, die alle ihren Weg fanden und dabei auch noch anderen Wegbegleitung und Unterstützung geleistet. Sie hatte viele Jahre in einer Genossenschaft Kredite vermittelt und Konten geführt und dabei manches Mal Rat und Ermutigung gratis dreingegeben. Sie hatte ihrem Mann den Rücken gestärkt, auch in kargen Nachkriegszeiten. Sie sang gern und half anderen dabei, durch Orgelspiel und Anleitung.

Sie löste durch klare Ansagen auch Reibung aus, aber als Mutter und Großmutter war sie dennoch oder vielleicht gerade deshalb unumstritten, und ihre Strenge war als Variante von Liebe und Ermutigung durchschaut und geachtet. Ihren Mann musste sie begraben, als er gerade in den Ruhestand gegangen war.

In ihren Achtzigern stellten sich allerlei Gebrechen ein, die sie klaglos ertrug. Als sie ihren Kindern endlich den Wunsch nach gründlicher medizinischer Abklärung erfüllte, kam dabei eine lange Liste bedrohlicher Erkrankungen heraus. Am ärgsten: Die zusammengesinterte Wirbelsäule hatte die ersten Nervenwurzeln gelähmt, Querschnittslähmung drohte. Die Veränderungen an den Adern und am Herzen waren für viele Arten schlimmer Ereignisse gut. Eine leitliniengerechte Prävention wies sie zurück, mit bayerisch-rauen Sätzen: „Der Herrgott weiß mehr als die Medizin«.

Ihren Glauben hatte sie immer konkret und praktisch verstanden und gelebt. Das tägliche Gebet und der Sonntagsgottesdienst waren ihr selbstverständlich, und sie versuchte, das Engagement für andere als Lebenshaltung weiterzugeben, wo sie konnte. Ihre große Sorge war, durch Krankheit zum Pflegefall zu werden und auf die Fürsorge anderer angewiesen zu sein. Über ihr Gebet in diesem Anliegen und um eine gute Sterbestunde sprach sie verstörend offen, und die 
Kinder und Enkel intervenierten oft: "Mach langsam, bleib noch ein bisschen! «

Die Feier zum 90. Geburtstag war ein großes Fest, natürlich gegen ihren anfänglichen Widerstand: "Kinder, das braucht's doch nicht.» Wenig später zog sie sich einen Magen-Darm-Infekt zu, von dem sie sich aber mit hausärztlicher Hilfe binnen weniger Tage erholte.

Am Tag nach sichtlicher Widerkehr der Kräfte legte sie sich nach dem Frühstück wie immer noch ein wenig hin. Eine Stunde später sah ihr Sohn nach ihr. Da war sie schon friedlich eingeschlafen und ohne Puls und Atmung.

Das Besondere war: In Bayern betet man, früher viel und heute wenig, besonders zum Heiligen Josef um eine gute Sterbestunde. M. hatte das auch getan, seit langer Zeit und regelmäßig. Sie starb im hohen Alter ohne große Schmerzen oder Atemnot, ohne Querschnittslähmung und Schlaganfall, auf eigenen Füßen gehfähig und bei klarem Verstand. Gegen alle Sorgen und Empfehlungen der Mediziner. Daheim. Und zwar am Josefstag, dem 19. März.

Falls jemand Konsequenzen daraus ziehen möchte, ein paar Möglichkeiten:

Gebete um eine gute Sterbestunde könnten - diesseits oder jenseits der Grenzen von Physiologie und Psychologie - sinnvoll sein, allen Zweifeln und allem Spott zum Trotz.

Selbst im größten Leid scheint der Schöpfer, wenn es einen gibt, gelegentlich zu starken, fast plakativen Signalen zu greifen.

Pietät und Respekt vor dem Leid der Zurückgelassenen verbieten diese Art von Überlegungen in aller Regel. Aber Hand aufs Herz: Manchmal lässt sich beim Sterben barmherziger göttlicher Humor nicht wirklich ausschließen, oder?

- Ottmar Leidner, Professor für Rehabilitationsmedizin, SRH Fachhochschule für Gesundheit Gera, seit 35 Jahren verheiratet und in der katholischen Kirche aktiv, u.a. in der GCL, einer geistlichen Gemeinschaft, die sich an der Spiritualität des Jesuitenordens orientiert. 